\title{
COMBINATION OF INSPECTION AND MONITORING TECHNIQUES FOR THE DETECTION OF FRACTURES IN CONCRETE WITH SELF-HEALING PROPERTIES
}

\author{
CHRISTIAN U. GROSSE ${ }^{*}$, FABIAN MALM ${ }^{\dagger}$ \\ "Technische Universitaet Muenchen \\ Muenchen, Germany \\ e-mail: grosse@tum.de \\ ${ }^{\dagger}$ Technische Universitaet Muenchen \\ Muenchen, Germany \\ e-mail: fabian.malm@tum.de
}

Key words: NDT, fractures, self-healing, acoustic emission, ultrasound, vibration analysis

\begin{abstract}
For applications studying fracture mechanical properties of concrete or the in-situ performance of cementitious materials non-destructive testing (NDT) and monitoring techniques (structural health monitoring, SHM) are beneficial. This will be demonstrated for a special application developing concrete exhibiting properties to heal or seal cracks (Jonkers 2011, Van Tittelboom et al. 2011). A development of a new material is typically done starting on a laboratory scale and ending up with demonstrating the techniques at real constructions. For the particular development - selfhealing concrete - it was found that a combination of inspection and monitoring techniques is most useful. This includes ultrasonic (UT), vibrational testing and acoustic emission techniques (AET). With AET (Grosse \& Ohtsu 2008) can the activation of healing agents be monitored as well as the opening of cracks under load. Since this technique is time consuming and laborious more efficient NDT techniques are required to help proving the performance of the new material to the stakeholder on the construction site. This could be ultrasonic or vibration testing.

The development and application of NDT inspection and monitoring techniques will be described as well as the combination of these techniques with simulations and material developments.
\end{abstract}

\section{INTRODUCTION}

Aspects of the application of cementitious materials in civil engineering include issues of sustainability and lifetime prognosis of components. To increase the performance of these components smart materials are used and modern non-destructive testing techniques can be applied to inspect and monitor their properties. The large variety of concrete mixes used in practice is leading to a different behavior concerning the applicability of NDT.
Moreover, a mixture out of the same ingredients (qualitatively and quantitatively) can perform differently due to the boundary condition during casting (temperature, segregation, compaction, etc.). In addition is concrete usually reinforced using steel bars or fibers. Therefore, it is not always easy to select the most useful NDT technique of example to detect the presence and geometry of cracks in concrete and in most cases, a combination of different techniques is required. This will be demonstrated for a very special application. 


\subsection{The HealCon project}

While autogenous healing mechanisms were intensively investigated earlier (Yang et al. 2009; Edvardsen 1996; Jooß 1999) it became obvious that these mechanisms are able to heal relatively small cracks only. In an international project sponsored by the European Commission in the $7^{\text {th }}$ framework programme three different healing or sealing mechanisms were investigated being applied for larger cracks and different crack types. The project is called HealCon: Self-healing concrete to create durable and sustainable concrete structures, http://www.healcon.eu/. For early age cracks, a non-elastic repair material can be proposed, such as calcium carbonate precipitated by bacteria (Jonkers 2011) or new cement hydrates of which the formation is stimulated by the presence of hydrogels. For cracks under dynamic load, elastic polymeric healing agents (polyurethane PUR or Epoxy resin based) are suggested that are encapsulated in microcapsules (Van Tittelboom et al. 2011). During the performance of cracks in the matrix, these capsules break releasing the polymeric healing agent what is sealing the crack and can lead to a strength regain.

There are two major tasks for NDT methods to be applied in this environment. During the development of a new material with selfhealing abilities, it is essential to get as much information as possible about its performance to design new mixes properly. Non-destructive testing has the potential to evaluate fractures in concrete but also monitor the release of healing agents or the loss and regain of properties including gas or water tightness.

For the in-situ application of the new material additional tasks consist of a proof of activation of the healing in larger components and the determination of the healing efficiency. A stakeholder would invest in such a new material only, if the effective operation of healing is verified.

During the HealCon project the best candidates among the non-destructive testing methods are investigated to be applied in small and large laboratory experiments as well as at real structures in-situ. This addresses also issues of structural health monitoring used for example to monitor the healing effects on a long-term basis and to assess the condition of the structure, where self-healing techniques are applied. In particular, techniques like ultrasound, RADAR, infrared-thermography, microwave, vibration and acoustic emission testing are candidates as well as several other techniques like CT scanning (for lab tests only), fiber optics or displacement field mapping. Other groups using ultrasound (In et al. 2013), resonance frequency (Yang et al. 2009) and acoustic emission techniques (Granger et al. 2007; Tsangouri et al. 2013) investigated already some of these methods. Additional research is necessary to transform these results and own developments into field applications. These own developments are based on NDT studies (Van Tittelboom et al. 2012; Grosse et al. 2013; Malm \& Grosse 2014; Flohr et al. 2015; Malm \& Grosse 2015; Gruyaert et al. 2015) and on acoustic emission techniques developed even earlier (Grosse \& Ohtsu 2008). However, since there are many data and comprehensive investigations including NDT were performed only some selected results can be presented in the following.

\section{SETUP OF LAB EXPERIMENTS}

After some small-scale lab experiments (Malm \& Grosse 2014), investigations using larger concrete beams under three-point bending applying different NDT techniques were performed. The purpose of these component tests was to study the activation of healing, to support the design and to develop further the NDT techniques under controlled conditions. A combination of ultrasonic, vibration analysis and acoustic emission (AE) techniques was implemented. For these tests, beams with dimensions of $1500 \times 1500 \times 5500 \mathrm{~mm}$ were casted according to Fig. 1. A set of control group specimens were concreted as well as some with the same geometry and encapsulated healing agents. For these preliminary experiments capillaries of relatively large size were used, while during the project much smaller capsules are about to be developed for later field applications. 


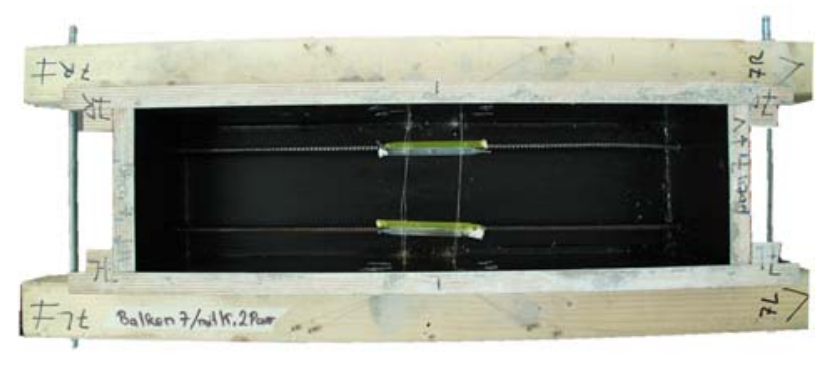

Fig. 1: Top view of a mould for the concrete beams with encapsuled self-healing agents ( 2 pairs of capillaries).

A various amount of glass capillaries with a diameter of $4 \mathrm{~mm}$ and filled with PUR, have been placed in the middle section of the test specimens using holders of thin steel wires concerning the here described experiments. In order to minimize the reaction time of the PUR an equal number of additional capillaries filled with water and accelerator were added. To create $300-350 \mu \mathrm{m}$ wide remaining cracks, the reinforced concrete specimens were loaded in crack-width-controlled three-point bending experiments. A notch at the bottom served as a predetermined breaking point. After loading, the specimens were stored in a climate chamber $\left(65 \%\right.$ relative humidity, temperature: $\left.20^{\circ} \mathrm{C}\right)$ for seven days to guarantee similar conditions for the curing process. During subsequent experiments, reloading was done in a way as described above.

\section{NDT SETUP AND RESULTS OF LAB EXPERIMENTS}

Prior to initial loading and reloading, $\mathrm{AE}$ sensors (Fig. 2) were attached to record AE data. During most experiments, ultrasonic measurements in through-transmission have been performed and vibrational data were recorded between the individual load cycles.

\subsection{Acoustic emission testing}

Acoustic emission analysis enables for monitoring microstructural changes like cracking and friction due to the emitted elastic waves. This technique is similar to recording earthquakes in seismology. A 16-channel transient recorder was used as an $\mathrm{A} / \mathrm{D}$ converter to record the data and to store full $\mathrm{AE}$ waveforms. The beams have been reinforced in the upper part (Fig. 2) to avoid sudden failure and the experiment was controlled using a crack mouth opening displacement sensor. The location of the 15 acoustic emission sensors surrounding the notched area in the middle of the beam are depicted in Fig. 2 as well as the ultrasound emitter and receiver at the outer sides left and right.

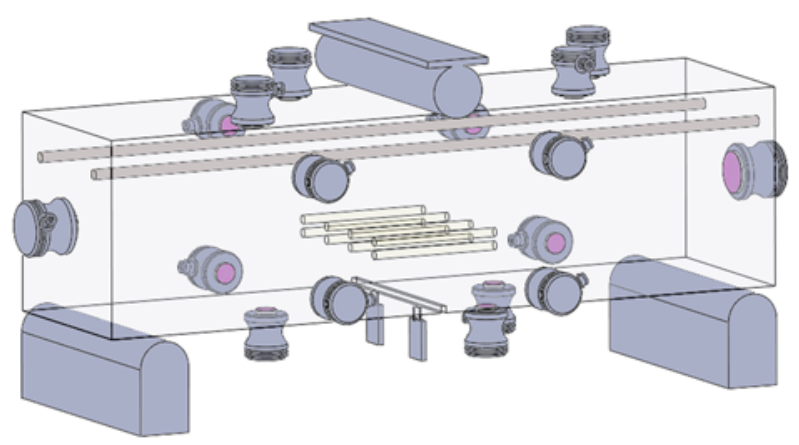

Fig. 2: Drawing representing a concrete beam with encapsulated self-healing agents (4 pairs of capillaries in the middle of the beam)

The AE activity during the initial and final loading is shown in the two graphs of Fig, 3 for the reference specimen ("REF") and the two graphs of Fig. 4 for a concrete specimen with encapsulated polymeric healing agents ("PUR") along with loading curves.
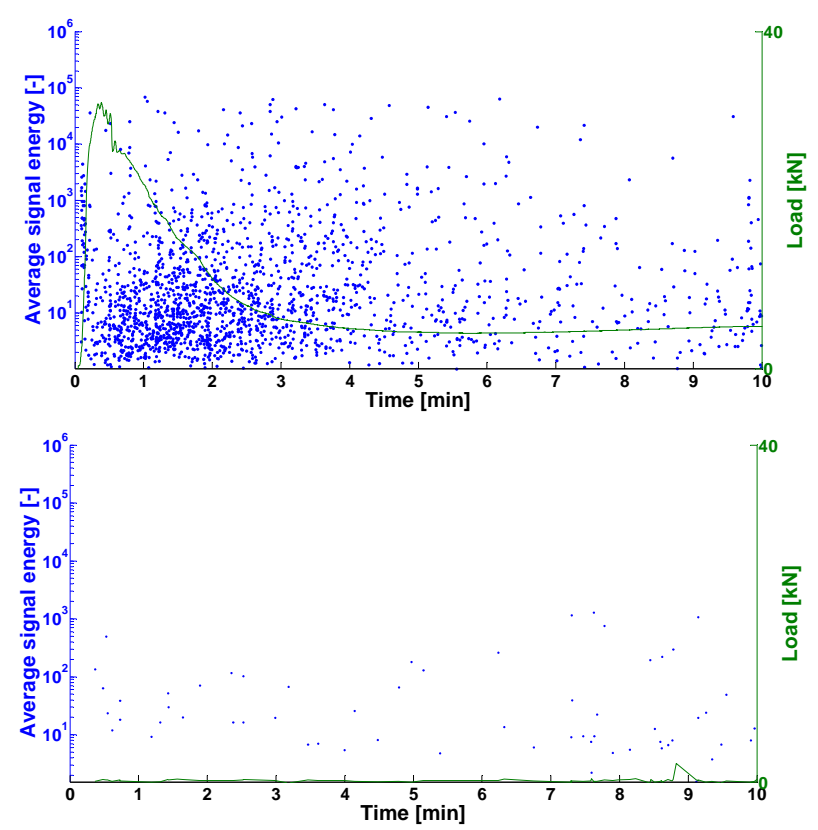

Fig. 3: REF specimen: initial cracking of the specimen (top) and re-cracking after reload (bottom) 

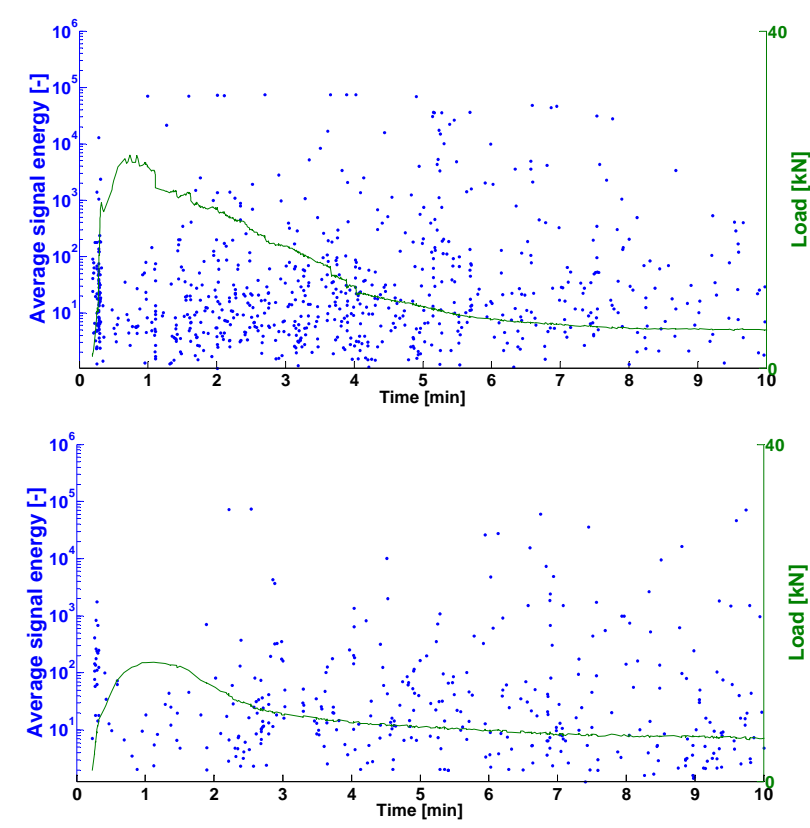

Fig. 4: PUR specimen (4 pairs of capillaries with embedded healing agents): initial cracking of the sound specimen (top) and re-cracking after reload (bottom)

During initial loading (upper graphs), many AEs occur with high energies in the peak and post-peak period due to fracture processes.
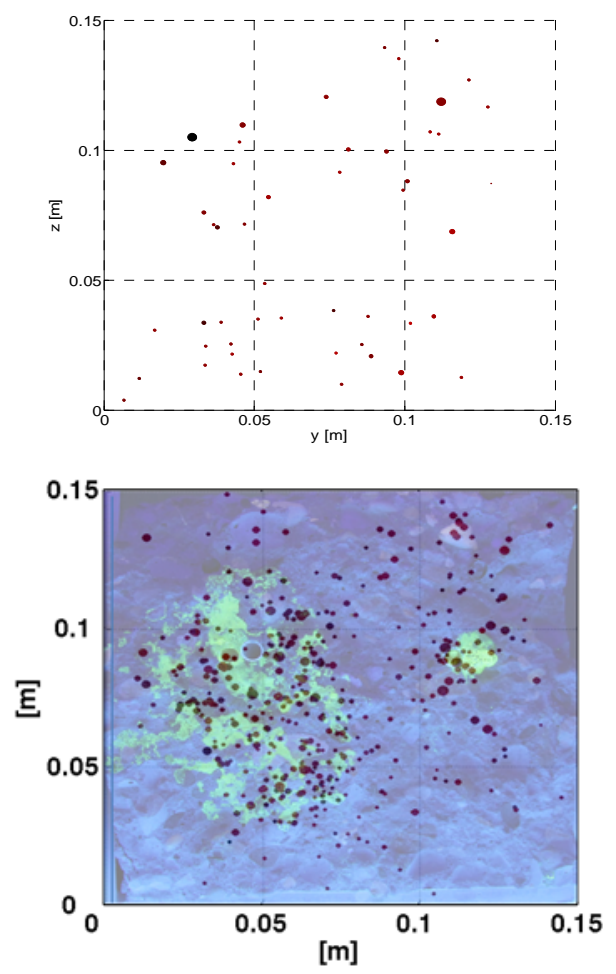

Fig. 5: Cross-section view of localized AEs (red dots) of REF specimen (top) and PUR specimen (2 pairs of capillaries, bottom) after curing 7 days in a climate chamber; the fluorescent area indicates the polymeric distribution
During reload the reference specimen exhibit no strength regain and only a few AEs with lower energies are observed (Fig. 3, bottom). The PUR specimen instead reveals a regain in strength and an increased number of AEs with higher energies (Fig. 4) compared to REF. A localization of the AEs of the PUR sample (Fig. 5, top) confirms that the emitted elastic waves emerge from polymeric fractures at the interface. Fluorescent pigments trace the distribution of the polymers after fracturing to make them more visible (Fig. 5, bottom).
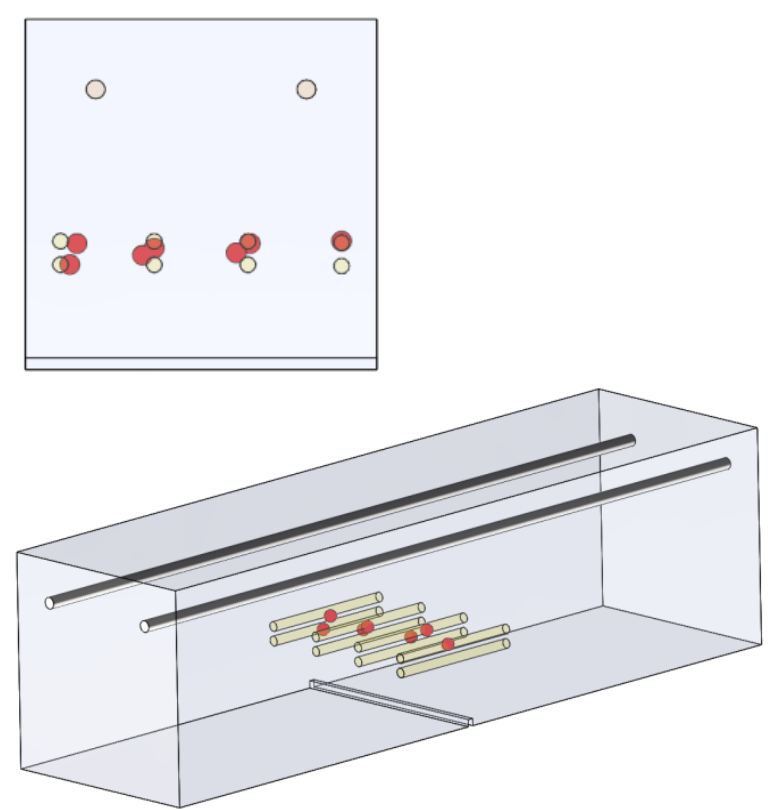

Fig. 6: View (cross-section - top, sideview - bottom) of selected localized $\mathrm{AE}$ representing breaks of embedded glass capillaries.

A few AE events with significantly higher energy compared to the majority of events occurred during the first loading cycle. The Localization of these events indicates that they correspond to the location of the capsules representing their breakage (Fig. 6).

\subsection{Ultrasound}

Since AE techniques are difficult being applied at real-world structures ultrasound methods and vibration analysis were further developed. This enables for a characterization of structural components at each scale concerning their mechanical properties. These will provide information about wave velocities, elastic moduli, and about other material 
parameters like porosity and crack density. The focus is here on a more averaged way to characterize material properties in a structural element.

Ultrasound transmission and reflection methods will help to evaluate the healing efficiency by the observation of changes of the velocity of the compressional and shear waves propagating through a specimen, and their amplitude and frequency content. They can help characterize the healing efficiency by investigating changes of e.g. the Young's modulus.

In the experiments described, ultrasonic measurements in through-transmission have been performed before, during and after loading and the data were evaluated in respect to their travel time and amplitude.

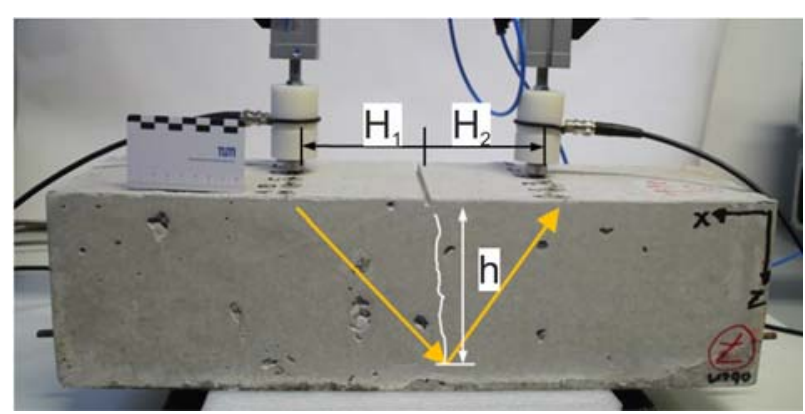

Fig. 7: Schematic drawing of the determination of the crack depth with ultrasound, where the yellow lines represent a possible travel path after cracking

By using the assumption of a straight travel path of the compressional wave for example, differences in the onset time allow for a calculation of the crack depth (Fig. 7):

$$
d=\sqrt{\left[\frac{\left(v_{\mathrm{p}} * \Delta t\right)^{2}+H_{1}-H_{2}}{2 * v_{\mathrm{p}} * \Delta t}\right]^{2}-H_{1}{ }^{2}} \quad[\mathrm{~m}]
$$

with: $\quad v_{p}$ : P-wave velocity - sound specimen $[\mathrm{m} / \mathrm{s}]$ $\Delta t$ : Time delay of p-wave due to cracking/healing [s]

$H_{1}$ : Distance - crack and transmitter [m]

$\mathrm{H}_{2}$ : Distance - crack and receiver [m] $d$ : crack depth $[\mathrm{m}]$

Therefore, an exact onset time determination is required to determine the crack depth precisely.

Preliminary test with predefined artificial cracks in similar concrete beams confirmed the applicability of this approach helping to determine the detectability and precision.

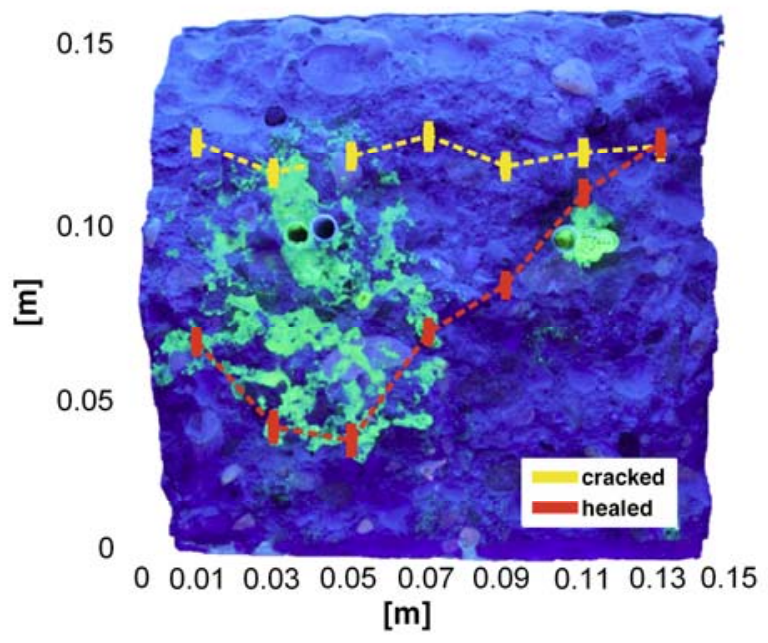

Fig. 8: Cross-section: Determined crack depth after cracking (blue) and after curing of the PUR (green)

Fig. 8 displays (yellow curve) the calculated crack depth as a result of delayed onset times on seven parallel transmission paths (0.01$0.13 \mathrm{~m}$ ) along the cross-section. The threepoint bending causes a nearly monotonic crack line along the specimen's width. Due to the wedge-shaped crack fracture a clearly visible determination of the final crack tip is impossible. After seven days of curing the ultrasonic transmission measurements were repeated (red curve). The polymer filling finally leads to a rebond of the crack flanks identified by shortened time delays of the propagating acoustic waves. Subsequently, the crack depth decreases at these locations where the polymer flows and hardens.

\subsection{Vibrational testing}

With vibrational test methods, the average mechanical properties of specimens and structural components can be determined. This method has the potential -like ultrasound - to be applied for a structural health monitoring.

The setup for vibrational testing for a typical measurement using the specimen mentioned in section 2 is shown in Fig. 9 where the instrumented impulse hammer is visible at the right and the modal sensor at the left side. 


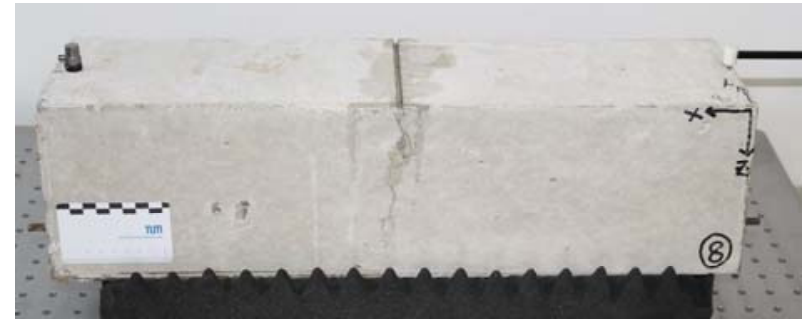

Fig. 9: Determination of the dynamic flexural modulus vibration analysis

Similar to ultrasonic testing measurements at three different states have been performed to determine the healing efficiency: initial, cracked and healed. By the mechanical excitation of the entire concrete beam using e.g. an instrumented vibration hammer, resonance frequencies help to determine the longitudinal, shear and flexural elasticity moduli. In the cracked state all moduli decrease compared to the initial state due to the weakening of the structure.

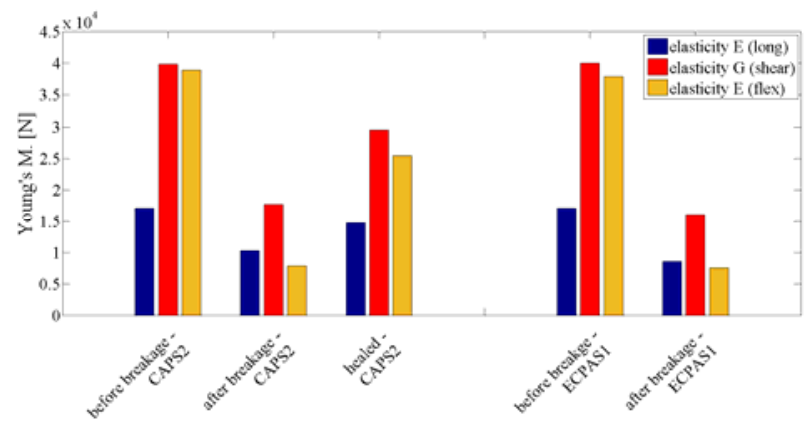

Fig. 10: Determination of the dynamic longitudinal, shear and flexural modulus with vibration analysis

After curing, all moduli increase again, where the flexural modes exhibit the steepest increase due to the effect that their vibrational mode is influenced utmost by the bending procedure. A control specimen with empty glass capillaries (ECAPS1) validated the results (Fig. 10).

\section{COMPARISON OF THE RESULTS AND OUTLOOK}

\subsection{NDT results}

This paper deals with three different NDT methods to determine the healing efficiency of lab-scale reinforced concrete beams with embedded polymeric precursors. Using $\mathrm{AE}$ analysis, the spatio-temporal development of fracture processes can be monitored. The size of the rebonded (glued) crack flanks and the quality of the bond are responsible for the amount of strength regain. In this way, the AE technique is able to observe these processes in detail. However, the precise localization of the acoustic events depends essentially on the sensor placement and the sensitivity of the recording equipment. As a consequence, $\mathrm{AE}$ techniques are limited to lab-scale tests. Ultrasonic transmission techniques are more flexible to be applied at large structures. Ultrasound allows for a characterization of structures even with one-sided access only and in a fast and practicable way. An accurate detection of the onset time of the acoustic waves and reproducible sensor coupling is required. Absolute quantitative measurements are difficult, if no long-term monitoring is established and the crack locations are unknown. Besides onset times and velocities are waveforms and energies useful interesting parameters to proof the healing efficiency but still need more investigations. Vibrational testing is giving a rough estimation of the healing efficiency only but enables for longterm SHM. On a lab-scale excitation and signal recording are easy to handle and the determination of the dynamic properties is done with high reproducibility. On the other hand need vibrational testing on a component or structure level certainly more evaluations and the sensitivity on a large scale is unknown yet.

\subsection{Further experiments}

In next step, the techniques will be applied to larger specimen representing full-scale components, like a slab and a column. These tests are performed soon and will be followed by applications at real constructions later on.

\section{ACKNOWLEDGEMENTS}

The European Commission, DG Research \& Innovation, partly supports the described work in the frame of the collaborative project HealCon (www.healcon.eu) via grant number 309451. The authors are thankful for the support by the HealCon partners and by their 
colleagues at the Center for Building Materials of the Technical University of Munich in particular Ms. Marina Nahm.

\section{REFERENCES}

Flohr K, Malm F, Grosse CU (2015) Schallemissionsanalyse zur Untersuchung der Effizienz von Selbstheilungsmechanismen in Beton, Proc. 20. Kolloquium Schallemission, DGZfP Berichtsband BB 153, June 2015, Garmisch-Partenkirchen, talk 13

Grosse CU, Ohtsu M (Eds.): Acoustic Emission Testing in Engineering - Basics and Applications. Springer publ., Heidelberg (2008), ISBN: 978-3-540-69895-1, 404 p.

Grosse CU, Van Tittelboom K, De Belie N (2013) Non-destructive testing techniques for the observation of healing effects in cementitious materials - an introduction. Proc. 4th International Conference on SelfHealing Materials - ICSHM 2013, Abstract ID No: CM-02

Gruyaert E, Feiteira J, Malm F, Tziviloglou E, Schlangen E, Grosse CU, De Belie N (2015) Non-destructive testing techniques to evaluate the healing efficiency of selfhealing concrete at lab-scale. Proc. $6^{\text {th }}$ International Conference on Emerging Technologies in Nondestructive Testing, Brussels, Belgium

In C-W, Holland RB, Kim J-K, Kurtis KE, Kahn LF, Jacobs LJ (2013) Monitoring and evaluation of self-healing in concrete using diffuse ultrasound. NDT\&E Int. 57, pp. 3644

Jonkers HM (2011) Bacteria-based self-healing concrete. Heron 56 (1/2), pp 1-12

Malm F, Grosse CU (2014) Examination of Rein-forced Concrete Beams with SelfHealing Properties by Acoustic Emission Analysis. Proc. $31^{\text {st }}$ Conference of the European Working Group on Acoustic Emission (EWGAE), Dresden, Germany

Malm F, Grosse CU (2015) Proof of efficiency: Examination of concrete beams with selfhealing properties by non-destructive testing methods. Proc. Fifth International
Conference on Self-Healing Materials (ICSHM2015), Durham, NC (U.S.A.), 2015 Tsangouri E, Aggelis DG, Van Tittelboom K, De Belie N, Van Hemelrijck D (2013) Detecting the Activation of a Self-Healing Mechanism in Concrete by Acoustic Emission and Digital Image Correlation, The Scientific World Journal, Article ID 424560

Van Tittelboom K, De Belie N, Van Loo D, Jacobs P (2011) Self-healing efficiency of cementitious materials containing tubular capsules filled with healing agent. Cement and Concrete Composites 33 (4), pp. 497505.

Van Tittelboom K, De Belie N, Lehmann F, Grosse CU (2012) Acoustic emission analysis for the quantification of autonomous crack healing in concrete. Construction and Building Materials 28 (1), pp. 333-341

Yang Y, Lepech MD, Yang E-H, Li VC (2009) Autogenous healing of engineered cementitious composites under wet-dry cycles. Cement and Concr. Res. 39 (5), pp. 382-390 